\title{
The Thing With Thingness
}

\section{JACQUELINE SHAW}

Rhode Island School of Design
"Details ... can be regarded as the minimal units of signification in the architectural production of meanings"1 states Marco Frascari in The Tell-The-Tale Detail. Within, he describes an additive presencing of an architecture through individual perception until the eventual "construction and construing ..." ${ }^{2}$ of the thing takes place. The sum of the parts equals the whole but/and embodies the essence of the whole simultaneously.

Some fifteen years later, the diagram is thrust forward as the ultimate but/and expression of the discipline. As stated by Peter Eisenman, "Generally, a diagram is a graphic shorthand. Though it is an ideogram, it is not necessarily an abstraction. It is a representation of something in that it is not the thing itself. In this sense, it cannot help but be embodied. It can never be value- or meaning-free even when it attempts to express relationships of formation and their processes. At the same time, a diagram is neither a structure nor an abstraction of structure. While it explains relationships in an architectural object, it is not isomorphic with it."3

If, however, one was to replace "diagram" with "detail" in the noted quotation, one can imagine Frascari and Eisenmann working on the same arithmetic equation from different ends - relatively the same, if only just a little different.

Generically, a [detail] is a graphic shorthand. Though it is an ideogram, it is not necessarily an abstraction. It is a representation of something in that it is not the thing itself. In this sense, it cannot help but be embodied. It can never be value- or meaning-free, even when it attempts to express relationships of formation and their processes. At the same time a [detail] is neither a structure nor an abstraction of structure. While it explains relationships in an architectural object, it is not isomorphic with it. ${ }^{4}$

The representation of details as a drawing practice has similarly remained relatively consistent over time. They describe, in a very specific language, an articulation of relationships between discreet objects and how those things come together to concretize an architectural element. The imaging of the detail, however, has adapted in order to keep up with the speed of contemporary image consumption. Imaged details are allowed to be evocative and speak more to an experience of the thing while unburdened by the weight of describing how it came into existence. One is internal while the other is external. The internal nature of the former is two-fold - proprietary capital and their primary role in the construction of the thing. The external, typically through the medium of photography, is universal in nature. What they project only needs to be construed rather than constructed ${ }^{5}$ and thus has the capacity to be repeatedly embodied and reinterpreted multiple times over.

This internal/external dynamic is an act of extreme expansion and contraction of experience which happens at the speed of a thumb swipe or scroll along a digital surface. Waffling between realities of construction economy and aesthetic, the close-cropped image of a carefully considered joint is required to be nimble, universal, and somehow concise in their expression. Simultaneously it must also embody process, personality, professional precision, and enough narrative for the "likes." When you do a search of a hashtag on Instagram of \#architecturedetail there are over 147,000 posts. ${ }^{6}$ Suppressed within this data set are the logistics of execution - specifications, particularities, site, histories, and inherent politics of the profession. Admittedly there are several layers of bias that are embedded within the algorithm based on individual interactions, histories, and high level so monitoring. In spite of these circumstances, the common denominator is they have all been defined by someone, somewhere, as an architecture detail.

In Kenneth Frampton's “Rappel A L'ordre: The Case for the Tectonic," the return to order, and in this case, the structural unit as tectonic, is presented as a disruption to the constant and ever presencing of imaging processes that currently render an architecture as eventually immaterial. "Rather than join in a recapitulation of avant-gardist tropes or enter into historicist pastiche or into the superfluous proliferation of sculptural gestures - all of which have an arbitrary dimension to the degree that they are based in neither structure nor in construction - we may return instead to the structural unit as the irreducible essence of architectural form."7

Leading one through an etymologic narrative of the word tectonic, Frampton illustrates “... a gradual passage from the ontological to the representational" 8 with a turn to Carlo Scarpa, similar to Frascari, as the end-all-be-all (i.e. tell-thetale) exemplar of the " 'thingness' of the constructed object. In "Carlo Scarpa and the Adoration of the Joint," Frampton situates the joint as the architectural generator over the plan " ... not only in respect of the whole but also with regard to alternative solutions lying latent, as it were, within any particular part." Describing his process with an heir of reverence: 

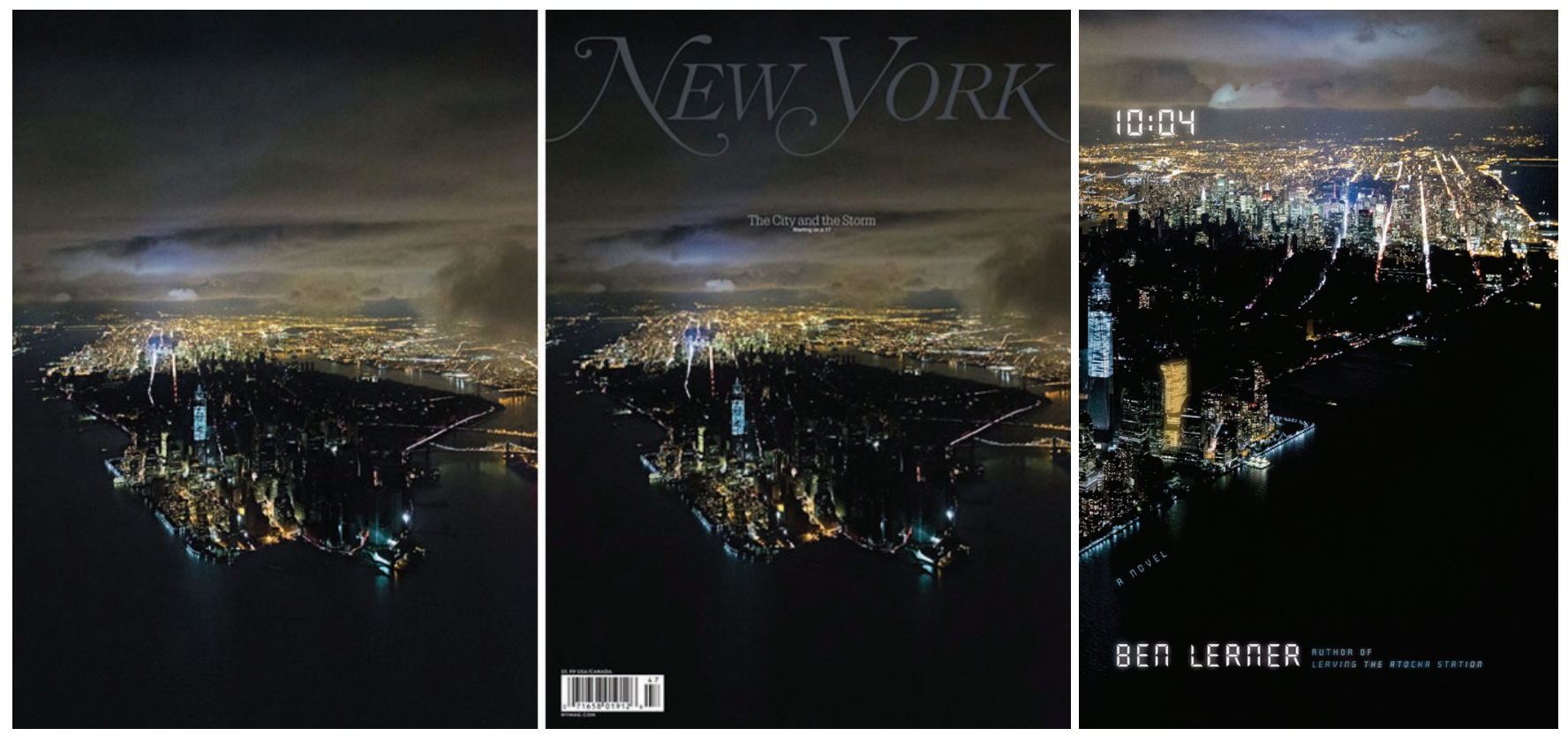

Figure 1. Left, Photo: Iwan Baan | New York Magazine. Nov 4, 2012; right, Lerner, Ben. 10:04. New York: Picador, 2014.

These alternatives arise spontaneously from Scarpa's method, his habit of drawing in relief, wherein an initial charcoal sketch on card, one of his famous cartoni, becomes progressively elaborated and overlaid by traces, washes, and even white-out to be followed by further delineations, entering into a cyclical process of erasure and redesign respect of a given junction, without ever fully abandoning the first incarnation of the solution. ${ }^{10}$

Frampton goes on to use Scarpa's gestural methods of making and his deep understanding of the constructed and the construed as the basis for the consideration of his practice. ${ }^{11}$ This resolute language gives light to a power structure that has conditioned a specific kind of thingness that is to be revered - one in which its existence alone is justification enough. The ability to recognize part to whole relationships in subtle (expansive) and obvious (contractive) ways is sufficient. The knowledge of why (the process of detailing) and how (the detail itself) will not and cannot be shared. Instagram further solidifies this power dynamic through dissemination producing an emotional reaction to imaged thingness as a conditioned reflex.

Accepting this framework, the questions center around acts of opposition and resistance. How does one dismantle a power structure that now uses image consumption and the speed that it occurs to maintain its agency? And in education how does one raise the bar of expectation from recognition to empowering students to ask questions of why and how something was done of themselves and their own work?

A close but not quite exact structural corollary to the creative and professional practices of the architect is of the writer. Multiple drafts followed by multiple edits, re-versioning processes that eventually, some many years later, could render itself acceptable for public consumption. Although potentially operating along similar timelines, contemporaneous creative writing practices differ in a particular way - the discipline that has capitalized on image culture and simultaneously implicated itself with little to no hesitancy. This is most clear in a genre of fiction writing in which the subjectivity of the novel aligns close to but is never quite identical with the subjectivity of the writer. It is one in which the author manages to leverage the culture of the close-cropped image, artfully edited social media feed without taking on the total weight of embodiment.

In 10:04, a novel by Ben Lerner, the reader is in a constant and active state of conflation. Caught between the personal narrative of the character, the author, and actual events from cultural and personal history, the novel takes place in New York in the years between Hurricanes Irene and Sandy. The novel is prefaced with a Hasidic belief that foregrounds simultaneously subjectivities that are just slightly different.

The Hassidim tell a story about a world to come that says everything there will be just as it is here. Just as our room is now, so it will be in the world to come; where our baby sleeps now, there too it will sleep in the other world. And the clothes we wear in this world, those too we will wear there. Everything will be as it is now, just a little different. ${ }^{12}$

This notion of an almost unrecognizable strangeness takes its first form as the cover art. Through the figures of One World Trade, and the headlights and stoplights forming the avenues, one begins to recognize the demarcation of Twenty-third street and the scene of the power outage in Manhattan post 
Hurricane Sandy. The longer one studies the image though, its strangeness becomes more apparent. The cover art is the Iwan Baan photograph that appeared on the cover of November 4, 2012 New York Magazine, mirrored, slightly skewed, and with a tighter crop. The same, just slightly different.

As an opening, the narrator, written in first person, describes a scene in which he, after a celebratory meal with his literary agent, experiences the sensation of his body being taken over by an ingested octopus. The scene takes place on the High Line specifically the cut which provides for the window overlooking traffic on Tenth avenue. What appears in the beginning as just over one page of text at exactly three hundred seventy-seven words takes formation as six pages of text, expanding upon specifics of the meal, layering in the context of a neighboring dinner table conversations, the apparent take-over of his body of the consumed octopus, and sensorial and spatial specifics of the walk to the site of the high line - all details for one's consideration.

The jump cut between the two passages provides operational tools for detailing. In the second passage, the reader experiences the active succession of images, sensations, memories and affects of the octopus takeover through the mathematic equivalences of the narrator's book advance laid out as a "strong six-figure" number in the first passage.

"A few months before, the agent had e-mailed me that she believed I could get a 'strong six-figure' advance based on a story of mine that had appeared in The New Yorker; all I had to do was promise to turn it into a novel,"13 becomes:

After my agent's percentage and taxes ... I would clear something like two hundred and seventy thousand dollars. Or Fifty-four IUIs, Or around four Hummer H2 SUVs. Or the two first editions on the market of Leaves of Grass. Or about twenty-five years of a Mexican migrant's labor, seven of Alex's in her current job. Or my rent, if I had rent control, for eleven years. Or thirty-six hundred flights of bluefin, assuming the species held. I swallowed and the majesty and murderous stupidity of it was all about me, coursing through me: the rhythm of artisanal Portuguese octopus fisheries coordinated with the rhythm of laborers' migration and the rise and fall of art commodities and tradable futures in the dark galleries outside the restaurant and the mercury and radiation levels of the sashimi and the chests of the beautiful people in the restaurant-coordinated, or so it appeared, by money. One big joke cycle. One big totaled prosody. ${ }^{14}$

The equating of two first-editions of Leaves of Grass and the twenty-five years wages of a single Mexican migrant worker (contextually and politically specific to the reader) with eleven years " ... of my rent if I had rent control ..." (specific to the world of the novel) provide an in, placing the familiar and known within an existence somewhere between fact and fiction. The world we know, just slightly different.

In a full unraveling and collapsing of the narrative, the essay "The Golden Vanity," by Ben Lerner was in fact published in the June 18, 2012 edition of The New Yorker. This excerpt appears as part two in the novel 10:04 through a rapid succession of the narrator describing a comedy of errors that is the submission-to-edit-to-publication process that may or may not have occurred for Ben Lerner in actuality. Somewhere between a clever parlor trick and mastery of future recognition, Lerner's use of the specific for the context to expand and contrast at will around, one questions what are the details of the reality encompassing the reader, the narrator, and the author? Are they all the same? And does it even matter? What one is left with is a sense of re-cognizing the familiar through the specific. Devices of speed, perception, repetition - all architectural - actively recast, rehash, and remix spatial occurrences. One possibility to begin, as presented by the novel, is the use of a singular repetitive entity:

'How exactly will you expand the story?' she'd asked, far look in her eyes because she was calculating tip. ${ }^{15}$

And allowing the follow-through to take a duplicitous form:

'I'll project myself into several futures simultaneously,' I should have said, a minor tremor in my hand; 'I'll work my way from irony to sincerity in the sinking city, a would-be Whitman of the vulnerable grid. ${ }^{\prime 16}$

'Like the princess in Sans Soleil, I'll make a long list of things that quicken the heart.' We emerged from the restaurant into the moving air. 'And you can be in it.' The streets were wet, but it wasn't raining now. We walked to the High Line entrance on Twenty-sixth and climbed the steps. The smell of viburnum, which either flower in winter or had flowered prematurely, mixed with the smell of car exhaust.

'I'm going to write a novel that dissolved into a poem about how the small-scale transformations of the erotic must be harnessed by the political.' Three-fifths of my neurons were in my arms as I touched each stand of sumac carefully placed among the disused rails. Never again would I eat octopus. ${ }^{17}$

\section{ENDNOTES}

1 Marco Frascari, "The Tell-the-Tale Detail," Semiotics, eds., John N. Deely and Margot D. Lenhart (New York: Springer Science and Business Media, 1981), $325-336$

2 Frascari, 325.

3 Peter Eisenman, "Diagram: An Original Scene of Writing," ANY: Architecture New York 23 (1998): 27-29.

4 Eisenman, 27, with edits by author.

5 Frascari, "The Tell-the-Tale Detail," 325-336. 
6 Instagram search performed by author on March 1, 2019.

7 Kenneth Frampton, "Rappel a l'ordre: The Case for the Tectonic," AD 60 (1990).

8 Frampton, "Rappel a l'ordre: The Case for the Tectonic."

9 Kenneth Frampton, "Carlo Scarpa and the Adoration of the Joint," Studies in Tectonic Culture: The Poetics of Construction in Nineteenth and Twentieth Century Architecture (Boston: The MIT Press, 2001), 307.

10 Frampton, "Carlo Scarpa and the Adoration of the Joint," 307.

11 Frampton, "Carlo Scarpa and the Adoration of the Joint," 307.

12 Ben Lerner, 10:04 (New York: Picador, 2014). Print, epigraph.

13 Lerner, 4.

14 Lerner, 155-156.

15 Lerner, 4, 158.

16 Lerner, 4.

17 Lerner, 158. 